\title{
Polymer-Coated Hydroxyapatite Nanoparticles for the Delivery of Statins
}

Thomas L Moore', Ann-Sofie Schreurs², Rachel A Morrison', Erika K Jelen ${ }^{1}$, Joachim Loo ${ }^{3}$, Ruth K Globus ${ }^{2 *}$ and Frank Alexis ${ }^{1,4 *}$

${ }^{1}$ Department of Bioengineering, Clemson University, Clemson, SC 29634, USA

'Space Biosciences Division, NASA Ames Research Center, Moffett Field, CA 94035, USA

${ }^{3}$ School of Materials Science and Engineering, Nanyang Technological University, Singapore 639798, Singapore

${ }^{4}$ Institute of Biological Interfaces of Engineering, Department of Bioengineering, Clemson University, Clemson, SC 29634, USA

\begin{abstract}
Statin drugs have been previously reported to increase the activity of osteoblasts, and are being investigated to treat low bone density pathologies. A controlled release nanoparticle drug delivery system may be used to prolong the exposure of osteoblasts to statin. Furthermore, nano-hydroxyapatite (nHA) has known osteoconductive and osteoinductive properties. The success of nanoparticle drug delivery systems is associated with safety, stability, biodegradability, and pharmacological parameters. nHA have been used as gene and drug delivery vehicles, however in vivo studies have shown that $\mathrm{nHA}$ aggregate, accumulate in the lungs, and impede the function of pulmonary surfactant. To address some of these challenges, we present a $\mathrm{nHA}$ coated with the biodegradable co-polymer poly(glycolide)poly(ethylene glycol) (PGA-PEG). PGA coating thickness could be precisely tuned by surface initiated ring opening polymerization to control the release of lovastatin. PGA-PEG coated nHA were shown to be internalized in human osteoblasts, and were also shown to maintain $70 \%$ cell viability in human umbilical vein endothelial cells at doses up to $500 \mu \mathrm{g} \mathrm{mL}^{-1}$ in vitro. Preliminary in vivo maximum tolerated dose studies showed that PGA-PEG coated $\mathrm{nHA}$ were less toxic compared to uncoated nHA. This hybrid polymer-HA nanoparticle drug delivery system has potential as a controlled release system for the delivery of statins to treat low bone density pathologies.
\end{abstract}

Keywords: Polymer-coated hydroxyapatite nanoparticles; Strains; Statin drugs; Polymerization

\section{Introduction}

Hydroxyapatite nanoparticles (nHA) have been investigated for numerous biomedical applications including implant coatings, tissue engineering scaffolds, in addition to gene and drug delivery systems [14]. In drug delivery, $\mathrm{nHA}$ are appealing due to drug loading and release through adsorption/electrostatic interactions, ability to tune nHA morphology, and ability to facilitate endosomal escape [4-6]. However, systemically delivered nHA has limited utility due to aggregation, and particle heterogeneity. Previously, nHA were shown to aggregate in vivo and be rapidly cleared by the liver $[7,8]$. nHA also were shown to block pulmonary capillaries, or inhibit the function of pulmonary surfactant $[9,10]$. Another challenge with nHA for drug delivery is that drug loading on $\mathrm{nHA}$ is generally limited to loading via adsorption or electrostatic interactions [4,11-14]. Here we developed a nHA drug delivery system with a multifunctional polymer coating to sequester and control the release of drugs (Figure 1).

Previous studies have investigated nHA for the delivery of anti-cancer drugs, antibiotics, genes, peptides, and bone growth drugs $[4,7,13-16]$. nHA can be loaded with charged molecules via adsorption or electrostatic interaction, and Kundu et al. [14] showed that doxorubicin, an anticancer drug, could load $30-50 \%$ onto the surface of nHA. Furthermore, Palazzo et al. [12] loaded alendronate, cisplatin, or di(ethylenediamineplatinum) medronate onto nHA via adsorption at $92 \%, 82 \%$, and $52 \%$ of drug adsorbed, respectively. Drugs or molecules could also be loaded via encapsulation into the calcium phosphate matrix. Bisht et al. [4] encapsulated plasmid DNA (pDNA) within calcium phosphate nanoparticles (NP) at $5.88 \mu \mathrm{g}$ per mg NP for intracellular pDNA delivery. Calcium phosphate NP have also been loaded with organic dyes via encapsulation, as a proof-of-concept for loading small, organic molecules [17]. Loading drug by adsorption to nHA surface may however result in potentially undesirable burst release of drug [18]. This could also result in changes to the physico-chemical properties of the NP, which may in turn affect the NP physiological behavior [19].
Use of a polymer coating may mitigate some of the challenges associated with nHA-mediated drug delivery, and polymeric nanoparticles have been previously investigated to treat bone pathologies [15,20,21]. Polymer coatings are able to encapsulate or bind drugs, prolong and tune release profiles, and mediate the interaction of the NP with the biological environment [15,21-24]. Shi et al. [22] reported microspheres of poly(lactide-co-glycolide) (PLGA) which encapsulated nHA and alendronate, a bisphosphonate drug used to treat bone diseases such as osteoporosis and Paget's disease. These microspheres, between 77 and $137 \mu \mathrm{m}$ in diameter, could sustain the release of alendronate over a 30-day period. Another study showed that while hydroxyapatite microspheres loaded with doxorubicin via adsorption exhibited burst release, PLGA-coated HA microspheres were able to sustain release for up to 7-days [23]. We report nHA coated with a poly(glycolide)-poly(ethylene glycol) (PGA-PEG) copolymer for the controlled release of lovastatin. As a proof of concept for using this PGA-PEG coated nHA drug delivery system (nHA-PGA-PEG), lovastatin was chosen as a potential therapeutic for the anabolic treatment of bone density-loss diseases. With nHA-PGA-PEG, the polymeric coating is able to encapsulate hydrophobic lovastatin within the PGA layer via hydrophobic-hydrophobic interactions. This loading approach could also be utilized to load other hydrophobic drugs.

*Corresponding authors: Frank Alexis, Department of Bioengineering, Clemson University, Clemson, SC 29634, USA, Tel: 864-656-3051; Fax: 864-656-4466; E-mail: falexis@clemson.edu

Ruth Globus, Space Biosciences Division, NASA Ames Research Center Moffett Field, CA 94035, USA, Tel: 864-656-3051; Fax: 864-656-4466; E-mail: ruth.k.globus@nasa.gov

Received September 01, 2014; Accepted October 15, 2014; Published October 24,2014

Citation: Moore TL, Schreurs AS, Morrison RA, Jelen EK, Loo J, et al. (2014) Polymer-Coated Hydroxyapatite Nanoparticles for the Delivery of Statins. J Nanomed Nanotechnol 5: 237. doi: 10.4172/2157-7439.1000237

Copyright: $\odot 2014$ Moore TL, et al. This is an open-access article distributed under the terms of the Creative Commons Attribution License, which permits unrestricted use, distribution, and reproduction in any medium, provided the original author and source are credited. 


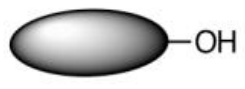

nHA
$+$

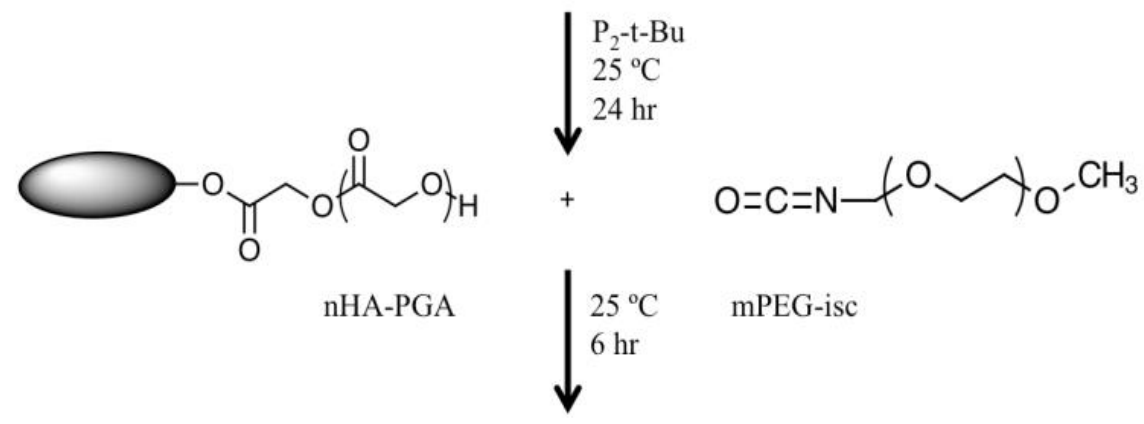<smiles>O=C1COC(=O)CO1</smiles>

Glycolide

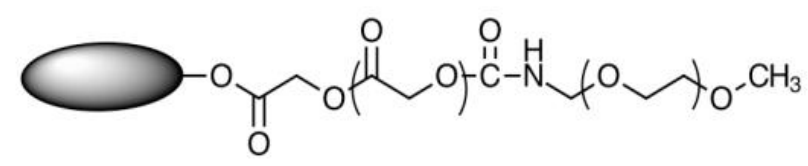

nHA-PGA-PEG

Figure 1: Schematic representation for the coating of $\mathrm{nHA}$ (grey ellipsoid) with $\mathrm{PGA}-\mathrm{PEG}$ using a one-pot, room temperature ring opening synthesis.

Furthermore, encapsulation also protects the drug from premature degradation, and controls release.

Statins are one class of drug shown to stimulate bone formation by enhancing the expression of bone morphogenic protein-2 (BMP-2) in osteoblasts $[25,26]$. In a culture of murine $(2 \mathrm{~T} 3)$ or human (MG63) bone cells, statins enhanced the expression of BMP-2 mRNA, increased the expression of type I collagen mRNA, lowered the expression of collagenase in MC3T3-E1 mouse osteoblast cells, and increase the expression of alkaline phosphatase [27]. Local controlled delivery of lovastatin at a fracture site using lovastatin microparticles has been shown to significantly improve fracture healing in mice [28], and controlled release of statins also improved osteogenic responses $[21,29,30]$. Thus, release kinetics and sustained exposure to lovastatin are important factors for the use of statins to treat bone pathologies. PLGA nanoparticles have previously been used to deliver lovastatin, and Ho et al. [21] reported PLGA particles that could deliver lovastatin over a 15-day period. Tai et al. [15] utilized PLGA nanoparticles co-loaded with simvastatin and nHA, and the nHA could tune the simvastatin release kinetics and adjust the $\mathrm{pH}$ of the microenvironment around the drug. Simvastatin could be released over a 14-day period.

Our study also utilized an outer PEG layer to stabilize the nanoparticle in an aqueous environment, as PEG has been shown to stabilize NP, reduce protein adsorption, and prolong the circulation time of nanocarriers in vivo [31,32]. Finally nHA, a primary component of bone tissue, has been shown to be osteoinductive and osteoconductive [33-38]. Thus, the nHA-PGA-PEG drug delivery system is designed such that the outer PEG shell stabilizes the NP in solution, the PGA coating sequesters hydrophobic lovastatin and controls its release, and the nHA core may further enhance osteoblast activity.

\section{Materials and Methods}

\section{Chemicals and reagents}

Nano-hydroxyapatite was purchased from SkySpring Nanomaterials, Inc. (Houston, TX, USA). Methoxy-poly(ethylene glycol)-isocyanate (mPEG-isc), MW 5000, was purchased from Nanocs, Inc. (New York, NY, USA). Glycolide, phosphazene base $\mathrm{P}_{2}-t$ $\mathrm{Bu}$ at $2 \mathrm{M}$ in THF, and all solvents were purchased from Sigma-Aldrich. Lovastatin was purchased from TCI America (Philadelphia, PA, USA). All materials were used as provided.

\section{PGA-PEG coating of nHA}

In a typical reaction, $40 \mathrm{mg}$ of $\mathrm{nHA}$ and $464 \mathrm{mg}(4 \mathrm{mmol})$ of glycolide were dried overnight under 32 in $\mathrm{Hg}$ vacuum. Glycolide and nHA were dissolved in $3 \mathrm{~mL}$ anhydrous dimethylformamide (DMF), and $\mathrm{P}_{2}-t-\mathrm{Bu}$ was added. The reaction vessel was purged under $\mathrm{N}_{2}$, and the reaction continued for $24 \mathrm{hr}$. PGA coating thickness was controlled by varying the ratio of $\mathrm{nHA}$ and glycolide. Next, $100 \mathrm{mg}(0.02 \mathrm{mmol})$ of $\mathrm{mPEG}$-isc was added directly into the reaction. This reaction continued under $\mathrm{N}_{2}$ for an additional $6 \mathrm{hr}$. The final product was washed twice with DMF and possible free monomer/polymer, and once with HyPure $\mathrm{H}_{2} \mathrm{O}$ via centrifugation at $5,000 \mathrm{rpm}$ for $5 \mathrm{~min}$. The pellet was then lyophilized and stored at $-20^{\circ} \mathrm{C}$ under $\mathrm{N}_{2}$ until used.

\section{Nanomaterial characterization}

Thermogravimetric analysis (TGA) was recorded on a TA Instruments Hi-Res TGA 2950 thermogravimetric analyser under $\mathrm{N}_{2}$ from $25^{\circ} \mathrm{C}$ to $600^{\circ} \mathrm{C}$ at $20^{\circ} \mathrm{C} \mathrm{min}^{-1}$. Transmission electron microscopy (TEM) was taken on a Hitachi H9500 high-resolution TEM at 300 $\mathrm{kV}$. Fourier transform infrared spectroscopy (FTIR) was recorded on a Thermo-Nicolet Magna 550 equipped with a Thermo-SpectraTech 
Foundation series Endurance Diamond ATR. IR: $2881 \mathrm{~cm}^{-1}\left(-\mathrm{CH}_{2}-\right.$ $\left.\mathrm{CH}_{2}-\mathrm{O}-\right), 1745 \mathrm{~cm}^{-1}(\mathrm{C}=\mathrm{O})$.

\section{In vitro release of lovastatin}

Release of lovastatin from nHA-PGA-PEG was determined using high performance liquid chromatography (HPLC). nHA nanoparticles coated with varying thicknesses of PGA were loaded with lovastatin using a solvent evaporation method. Briefly, particles were dissolved at $5 \mathrm{mg} \mathrm{mL}^{-1}$ in a solution of lovastatin in acetonitrile $\left(1 \mathrm{mg} \mathrm{mL}^{-1}\right)$. This NP solution was then dropped into $\mathrm{HyPure} \mathrm{H}_{2} \mathrm{O}$ at a $1: 2$ ratio and stirred for $2 \mathrm{hr}$. Lovastatin/NP was then washed three times in $\mathrm{HyPure} \mathrm{H}_{2} \mathrm{O}$ via centrifugation in $100 \mathrm{kD}$ MWCO centrifugal filter units at 3500 rpm for 5 minutes. $1 \mathrm{mg}$ of Lovastatin/NP was then loaded into the top of $3.5 \mathrm{kD}$ MWCO Slide-A-Lyzer MINI dialysis units. Dialysis units were stored in microcentrifuge tubes containing HyPure $\mathrm{H}_{2} \mathrm{O}$ at $37^{\circ} \mathrm{C}$. At each time point, the dialysate was collected and replaced with fresh $\mathrm{H}_{2} \mathrm{O}$. Dialysate was lyophilized and analysed for lovastatin content. HPLC was performed using a Waters 1525 Binary HPLC pump with a 2998 photodiode array detector. An Alltima C18 column $(4.6 \times 25 \mathrm{~mm}$, $5 \mu \mathrm{m}$ ) was used with a mobile phase of $71 \%$ acetonitrile (ACN) and $29 \%$ $0.5 \mathrm{M}$ ammonium acetate in $\mathrm{H}_{2} \mathrm{O}$ at $\mathrm{pH} 4.0$, and a flow rate of $1 \mathrm{~mL} \mathrm{~min}$ ${ }^{1}$. Lovastatin was detected at a wavelength of $238 \mathrm{~nm}$.

\section{Cell culture}

Human umbilical vein endothelial cells (HUVEC) and endothelial growth media (EGM) were purchased from Lonza (Walkersvill, MD, USA). Fetal bovine serum (FBS) was purchased from Atlanta Biologicals (Lawrenceville, GA, USA). HUVEC were grown on collagen-1 coated flasks in EGM supplemented with 20\% FBS. Bone marrow cells from 4-mo. old, male, $\mathrm{C} 57 \mathrm{Bl} / 6 \mathrm{~J}$ mice were flushed, then plated and grown in medium supplemented under conditions that induce osteoblast differentiation, as described previously [39].

\section{In vitro cytotoxicity}

HUVEC were seeded at 10,000 cells well ${ }^{-1}$ in a 96-well plate with five repeats per test concentration. Uncoated $\mathrm{nHA}$ and nHA-PGA-PEG were prepared by first dissolving at $5 \mathrm{mg} \mathrm{mL}^{-1}$ in acetonitrile (ACN). Next, the nHA or nHA-PGA-PEG solution was dropped at a 1:2 ratio into sterile HyPure $\mathrm{H}_{2} \mathrm{O}$ in an aseptic hood and stirred for $2 \mathrm{hr}$. NP were washed twice with $\mathrm{HyPure} \mathrm{H}_{2} \mathrm{O}$, and once with sterile filtered phosphate buffered saline (PBS) via centrifugation in a $100 \mathrm{kD}$ MWCO centrifugal filter unit. These separate solutions were then dispersed in EGM at varying concentrations and added to cells. HUVEC were incubated with nanomaterials for $24 \mathrm{hr}$ at $37^{\circ} \mathrm{C}$ and $5 \% \mathrm{CO}_{2}$. Media was removed, and cells were gently washed twice with sterile PBS.

A PrestoBlue cell viability assay (Life Technologies) was performed by mixing PrestoBlue reagent at a 1:9 ratio with EGM. $100 \mu \mathrm{L}$ of working solution was added to cells, and these were incubated protected from light for $45 \mathrm{~min}$ at $37{ }^{\circ} \mathrm{C}$ and $5 \% \mathrm{CO}_{2}$. Fluorescent intensity was measured with a BioTek Synergy 4 plate reader at $\lambda_{\text {ex }}$ of $560 \mathrm{~nm}$, and $\lambda_{\text {em }}$ of $590 \mathrm{~nm}$. Cell viability was determined by normalizing the fluorescent intensity of each test well to the average intensity of control cells treated with only EGM.

\section{In vitro cellular uptake}

Bone marrow cells grown under osteoblastogenic conditions were approximately $80 \%$ confluent after 10 days in culture. Alexa Fluor 532 was loaded into nHA-PGA-PEG via encapsulation following the lovastatin loading procedure. Multiple controls were included to ensure the specificity of nHA uptake and dye delivery as follows. The cells were imaged without any addition of the nHA or dye, or with the dye added alone or with nHA added alone. Cells were exposed to media only, media with fluorescent dye, or dye-loaded nHA-PGA-PEG. Fluorescent images were taken from $5 \mathrm{~min}$ to $72 \mathrm{hr}$ following exposure. Fluorescent microscopy was recorded on a Zeiss LSM 510 microscope.

\section{In vivo maximum tolerated dose}

All in vivo work was done in accordance with Clemson Institutional Animal Care and Use (IACUC) approved protocols. Animals were housed at Clemson University's Godley-Snell Research Center. BALB/c mice were treated with escalating doses of either uncoated nHA or nGP. Nanoparticles were prepared similar to the in vitro cytotoxicity studies, and systemically administered via tail vein injection. Mice were observed for behavioral signs of significant toxicity. Non-responsive behavior, or a mass loss of $15 \%$ of initial body weight was classified as toxic [40,41]. Mice were observed for 7 days following injection. Following the completion of the maximum tolerated dose study, mice were euthanized as per IACUC protocol requirements. Organs were fixed in $4 \%$ formaldehyde overnight and processed for histological analysis. Briefly, organs were embedded in paraffin, sectioned at $5 \mu \mathrm{m}$, and stained with hematoxylin and eosin (H\&E), or Alizarin red.

\section{Results and Discussion}

Bone density loss can be attributed to a number of pathological abnormalities. For example, microgravity-induced bone density loss is caused by the lack of mechanical stresses in space $[42,43]$. As previously stated, nHA has been investigated for drug delivery applications. However, loading is often limited to drug adsorption or electrostatic interactions. Also, nHA use as a systemic therapy is limited due to instability and aggregation. For bone applications though nHA is promising due to its osteoconductive and osteoinductive capabilities, and because it is a naturally occurring, primary component of bone $[33-36,38,44]$. Thus, this nHA-PGA-PEG delivery system is able to overcome the challenge of drug loading by encapsulating drug within the PGA, increasing stability with a PEG coating, and retaining the nHA core. The multifunctional nanoparticle could prolong the release of an osteoblast-stimulating drug (lovastatin), and enable long-term space flight or potentially treat other pathologies $[15,21,44]$.

\section{Nanoparticle synthesis and characterization}

nHA was coated by directly functionalizing the surface with poly(glycolide)-poly(ethylene glycol) (PGA-PEG). Hydroxyl groups within the crystal structure of nHA were utilized to initiate the ringopening polymerization of PGA directly on the nHA surface (nHAPGA) $[16,36,45,46]$. By controlling the ratio of the monomer glycolide $(\mathrm{M})$ and the catalyst $\mathrm{P}_{2}-t-\mathrm{Bu}(\mathrm{C})$, it was possible to change the thickness of the PGA coating. PEG was then coupled with the terminal hydroxyl groups on PGA chain ends by directly introducing isocyanatefunctionalized PEG into the reaction mixture. Figure 2 shows the chemical characterization of nHA coated with PGA-PEG (nHA-PGA$\mathrm{PEG}$ ). Figure 2A shows thermogravimetric analysis (TGA) of nHA, nHA-PGA, and nHA-PGA-PEG. There was no mass loss for nHA over a temperature range from $25^{\circ} \mathrm{C}$ to $600^{\circ} \mathrm{C}$, indicating no presence of organic polymer (Figure $2 \mathrm{~A}$ ). However, by varying the monomer to catalyst ratio (M/C) at 200,100 , and 50 , it was possible to achieve PGA coatings of 20,40 , and $60 \mathrm{wt} \%$, respectively. Moreover, by introducing PEG-isocyanate directly in a one-pot reaction it was possible to further functionalize PGA coated nHA with a layer of PEG (Figure 2B).

Fourier transform infrared (FTIR) spectroscopy of nHA, nHA- 
Citation: Moore TL, Schreurs AS, Morrison RA, Jelen EK, Loo J, et al. (2014) Polymer-Coated Hydroxyapatite Nanoparticles for the Delivery of Statins. J Nanomed Nanotechnol 5: 237. doi: 10.4172/2157-7439.1000237

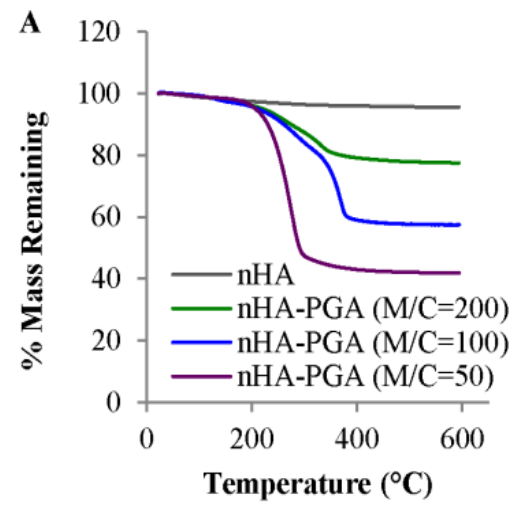

C
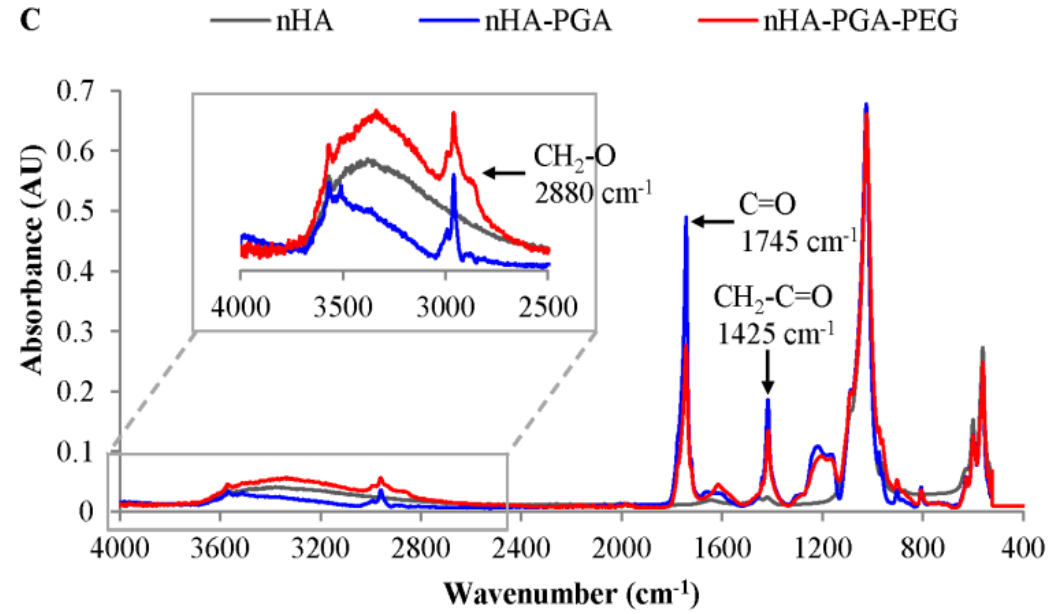

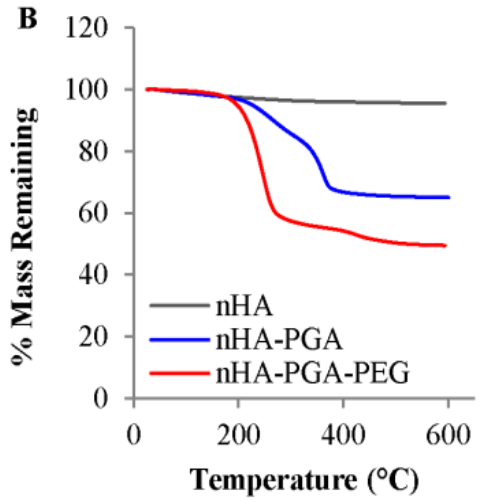

nHA-PGA-PEG

Figure 2: (A) Thermogravimetric analysis (TGA) of $n H A$ coated with various thicknesses of PGA. (B) TGA of uncoated $n H A$, $n H A$ coated with PGA (nHA-PGA) and nHA coated with PGA-PEG (nHA-PGA-PEG). (C) Fourier transform infrared spectroscopy of nHA, nHA-PGA, and nHA-PGA-PEG.
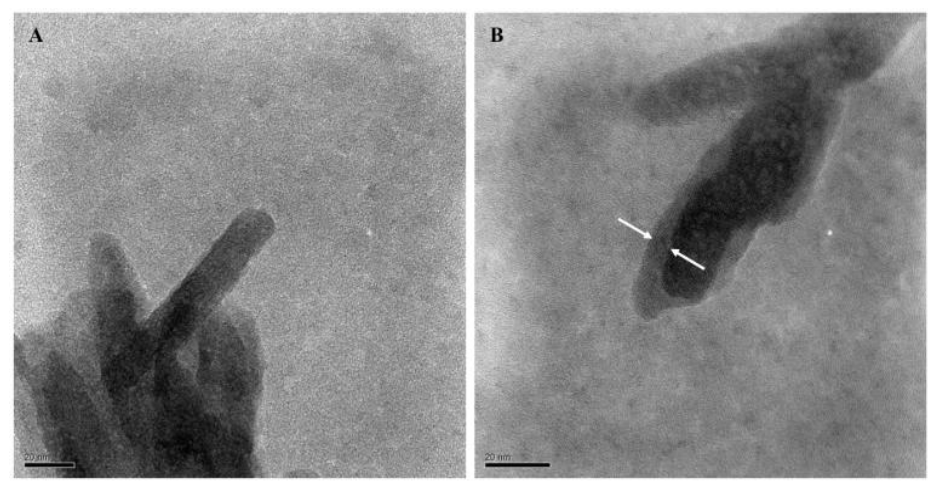

Figure 3: Transmission electron microscope images of uncoated (A) nHA, and (B) nHA-PGA-PEG. Polymer coated nHA-PGA-PEG showed contrast between the $\mathrm{nHA}$ crystals and the polymer coating, as indicated with the white arrows. Scale bars represent $20 \mathrm{~nm}$.

PGA, and nHA-PGA-PEG further verifies the presence of a polymer coating on nHA (Figure 2C). The carbonyl stretch at $1745 \mathrm{~cm}^{-1}$ is present in both nHA-PGA and nHA-PGA-PEG, and corresponds to the ester bond in PGA. The carbonyl group also results in $\mathrm{CH}_{2}-\mathrm{C}=\mathrm{O}$ bending at $1425 \mathrm{~cm}^{-1}$. In the nHA-PGA-PEG sample, there is a slight $\mathrm{CH}$ stretching vibration at $2880 \mathrm{~cm}^{-1}$ which corresponds to the ether bond in PEG (Figure 2C, inset) [47]. 

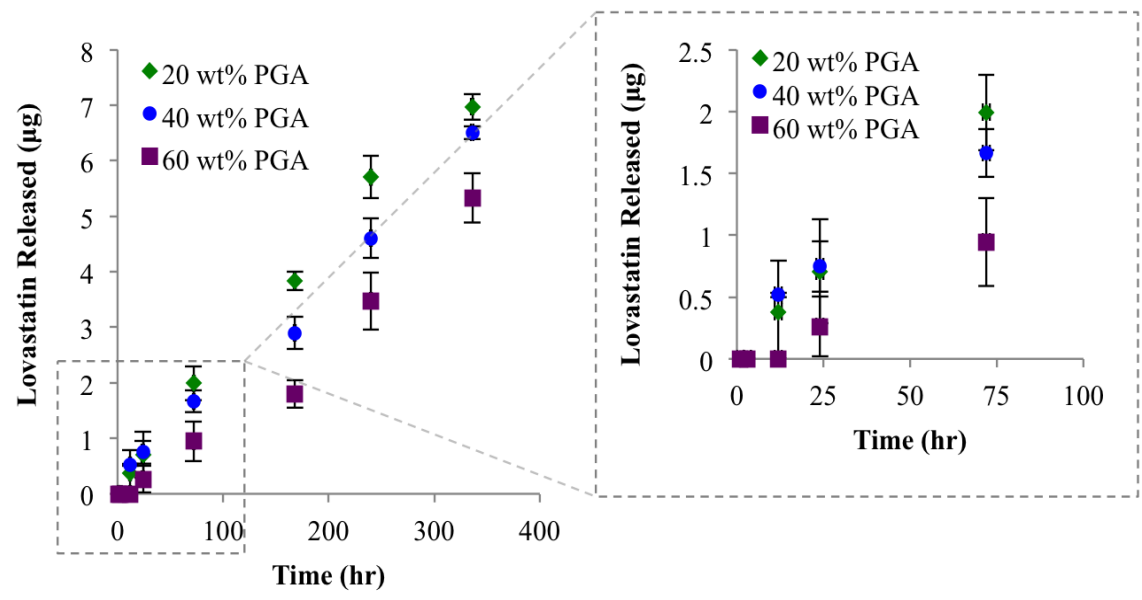

Figure 4: Release of lovastatin from nHA-PGA with varying thicknesses of PGA coating. PGA coating thickness controlled the release rate, with the thin coating (20 wt \%) releasing quickest, and the thicker coatings (40 and $60 \mathrm{wt} \%$ ) releasing slower. The magnified region shows the first $72 \mathrm{hr}$ of release, emphasizing the lag in release from the $60 \mathrm{wt} \% \mathrm{PGA}$ coated $\mathrm{nHA}$.

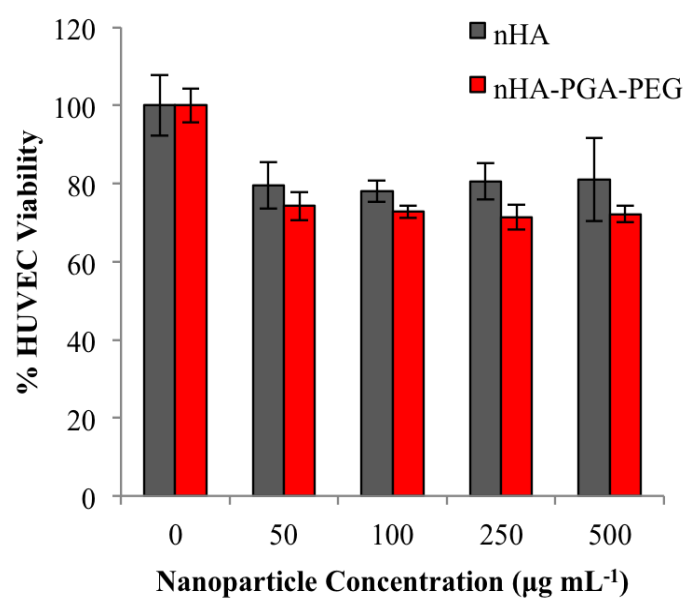

Figure 5: Cytotoxicity was measured in HUVEC with increasing doses of either nHA or nHA-PGA-PEG. No significant differences were noted between the two formulations, and cell viability remained at $80 \%$ compare to the control at concentrations as high as $500 \mathrm{\mu g} \mathrm{mL}^{-1}$.

\section{Lovastatin loading and release}

The rate of lovastatin release was controlled by varying the polymer coating thickness. Release from polymeric systems is controlled by a number of factors including polymer-drug interactions, polymer degradation and swelling, drug diffusivity through the polymer matrix, and polymer thickness [48-50]. Release from nHA-PGA was inversely related to polymer thickness: as polymer coating thickness increased, lovastatin release slowed. As drug diffusivity is controlled by factors such as polymer crystallinity, swelling, degradation kinetics, and thickness, it is expected that thicker polymers coatings will result in slower release kinetics. Figure 4 shows that release from 20 and $40 \mathrm{wt} \%$ PGA coated nHA was instantaneous, while there was an initial lag period of $24 \mathrm{hr}$ for lovastatin release from $60 \mathrm{wt} \%$ PGA. Moreover, $20 \mathrm{wt} \% \mathrm{nHA}-\mathrm{PGA}$ showed a 1.32 -fold increase in release compared to $60 \mathrm{wt} \%$ nHA-PGA after 14 days. While lovastatin is generally administered as an oral therapeutic, it however exhibits low oral bioavailability [51]. Several studies have investigated lovastatin for local injection to treat bone pathologies [21,28-30], however by encapsulating lovastatin within a NP drug delivery system it may be possible to improve the treatment of bone pathologies systemically by delivering the drug to multiple sites (Figure 5).

\section{In vitro toxicity and cellular uptake}

While all components of nHA-PGA-PEG are independently used clinically for biomedical applications, nanoparticle drug delivery systems must be robustly tested for safety of use. Here, we measured cytotoxicity in HUVEC after treating with increasing doses of either uncoated nHA, or nHA-PGA-PEG for $24 \mathrm{hr}$. Five repeats were used for each test, and data were analyzed using a Tukey's honestly significant difference test. Compared to the control, all formulations showed a slight decrease in cell viability. However, there was no significant difference in cytotoxicity from either uncoated or coated NP, and HUVEC maintained $70 \%$ viability at doses as high as $500 \mu \mathrm{g} \mathrm{mL}$ 1. Nanomaterial cytotoxicity is a factor of NP stability, size, shape, surface functionalization, method of synthesis, uptake, degradation, and material. These cytotoxicity data appear similar to other cell types in vitro, where human monocyte-derived macrophages showed $70 \%$ viability after treatment with rod-shaped $\mathrm{nHA}$ at up to $500 \mu \mathrm{g} \mathrm{mL}^{-1}$ [52]. Zhao et al. [53] tested the cytotoxicity of rod-shaped nHA in BEAS2B human bronchial epithelial cells, RAW264.7 mouse monocyte/ macrophage cells, and HepG2 human hepatocellular liver carcinoma cells. Cell viability was $>90 \%$ following exposure up to concentrations of $300 \mu \mathrm{g} \mathrm{mL} \mathrm{m}^{-1}$.

Primary bone marrow cells from adult mice were flushed and cultured in medium containing factors that promote osteoblastogenesis, and uptake studies were conducted to verify cellular internalization of nHA-PGA-PEG loaded with Alexa Fluor 532 (Figure 6). Some extracellular aggregation of nHA-PGA-PEG was observed, evident as intense fluorescence. These decreased over time and correlated with an increase of fluorescence localization inside the cells. The peak of intracellular fluorescence was reached in approximately 30-60 mins. Figure 7 shows a higher magnification to image the localization of the nHA-PGA-PEG within the cells. The fluorescence was clearly localized inside the cells, and seemed to concentrate into some organelles. These images were collected $24 \mathrm{hr}$ after addition of the NP and after the media 
Citation: Moore TL, Schreurs AS, Morrison RA, Jelen EK, Loo J, et al. (2014) Polymer-Coated Hydroxyapatite Nanoparticles for the Delivery of Statins. J Nanomed Nanotechnol 5: 237. doi: 10.4172/2157-7439.1000237

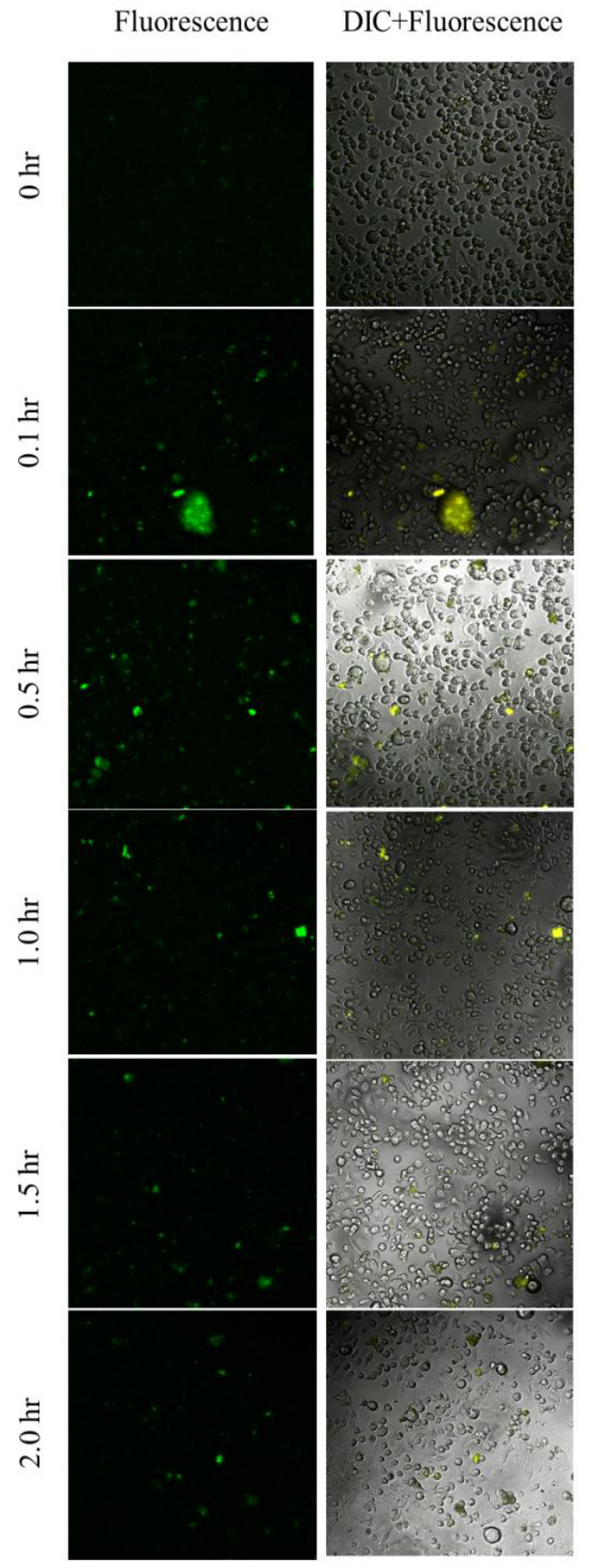

Figure 6: Cellular uptake of dye-loaded nHA-PGA-PEG was shown in primary bone marrow cells grown under conditions that promote osteoblast differentiation. Fluorescence microscopy (left) shows signal from the dye/NP. Overlay of fluorescence microscope images with differential interference (DIC) microscopy (right) shows the NP localized within cells.

was changed, thus to remove any remaining nHA-PGA-PEG. The fluorescence observed is specific to the cells.

\section{In vivo toxicity}

One limitation for the use of nHA as a systemic delivery system is instability that can lead to toxicity. Previous studies have shown that nHA can aggregate in blood and become localized in the lungs. nHA also has been shown to adversely affect pulmonary surfactant in the lungs
$[9,10]$. We hypothesized that the PGA-PEG coating therefore mediates the interaction of the NP with cells. Both PGA and PEG are FDAapproved materials frequently used in biomedical applications. PEG has also been shown to improve the aqueous stability of nanoparticles $[32,54,55]$. Thus, the polymer coating may reduce inherent cytotoxicity associated with nHA.

Preliminary maximum tolerated dose studies were performed in $\mathrm{BALB} / \mathrm{c}$ mice by injecting escalating doses of uncoated $\mathrm{nHA}$ or nHA-PGA-PEG. Mice were monitored for signs of morbidity (lack of mobility, $>15 \%$ loss of initial body mass), and were observed over $168 \mathrm{hr}$. Table 1 shows the survival time of mice injected with the two different formulations. Mice were able to tolerate higher doses of nHAPGA-PEG compared to nHA. This is potentially due to the polymer coating, particularly the PEG improving the dispersion of nHA in blood. Aoki et al. [9] found the medium lethal dose of intravenously administered nHA in Wistar rats to be $160 \mathrm{mg} \mathrm{kg}^{-1}$. The cause of morbidity in the rats was attributed to nHA aggregation and blockage of pulmonary capillaries. Our histological examination of mice treated with nHA at high doses show that this may also have been the cause of death for BALB/c mice treated with nHA (Figure 8). Tissues were stained with haematoxylin and eosin, or Alizarin red to show tissue structure or the presence of calcium, respectively. Morphologically, the saline injected lung tissue appeared healthy. H\&E micrographs clearly show the clean structure of healthy alveolar sacs. The tissue from the nHA injection however appeared severely inflamed with a high presence of immune cells. Furthermore there is significant aberration in the alveolar structure in the nHA lung tissue compared to the saline injection. Alizarin red staining for calcium showed healthy lung tissue (pink color) following the saline injection, while the nHA injection showed large calcium deposits, shown as red aggregates. Small deposits of nHA were also found in the brain, liver, and heart tissues. These data supports results by Aoki et al. [9] that the cause of morbidity from systemically administered nHA was aggregation and blockage of pulmonary capillaries.
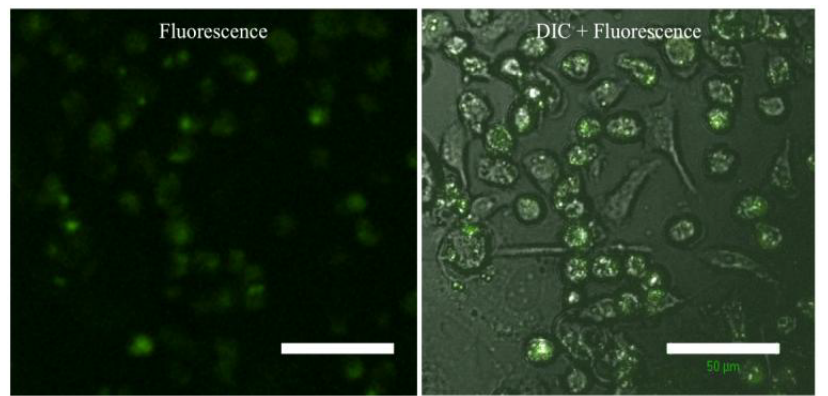

Figure 7: Magnified images of osteoblasts $24 \mathrm{hr}$ following exposure to dye-loaded nHA-PGA-PEG showed localization of NP in cells. Scale bars represent $50 \mu \mathrm{m}$.

\begin{tabular}{|c|c|c|}
\hline \multirow{2}{*}{ Dose $\left(\mathbf{m g ~} \mathbf{~ k g}^{-1}\right)$} & \multicolumn{2}{|c|}{ Survival Time (hr) } \\
\cline { 2 - 3 } & nHA & nHA-PGA-PEG \\
\hline $\mathbf{1 6 0}$ & 168.000 & 168.000 \\
\hline $\mathbf{2 0 0}$ & 0.030 & 168.000 \\
\hline $\mathbf{2 4 0}$ & 0.008 & 24.000 \\
\hline $\mathbf{2 8 0}$ & 0.008 & 24.000 \\
\hline
\end{tabular}

Table 1: Survival time of mice treated with uncoated or coated $\mathrm{nHA}$. 
Citation: Moore TL, Schreurs AS, Morrison RA, Jelen EK, Loo J, et al. (2014) Polymer-Coated Hydroxyapatite Nanoparticles for the Delivery of Statins. J Nanomed Nanotechnol 5: 237. doi: 10.4172/2157-7439.1000237

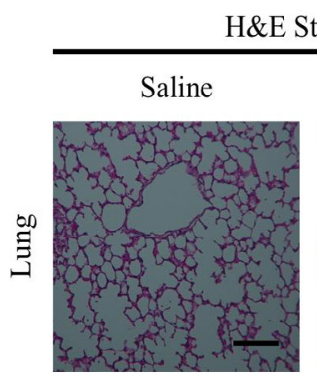

H\&E Staining
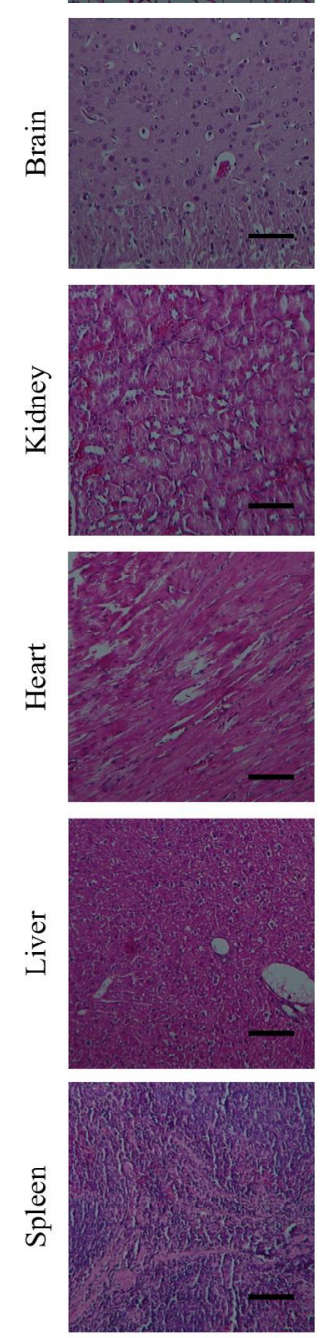

nHA
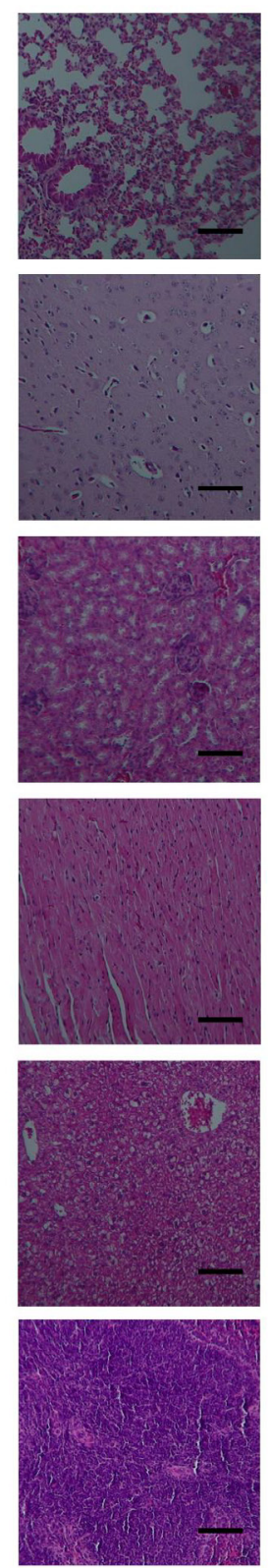

Alizerin Red Staining
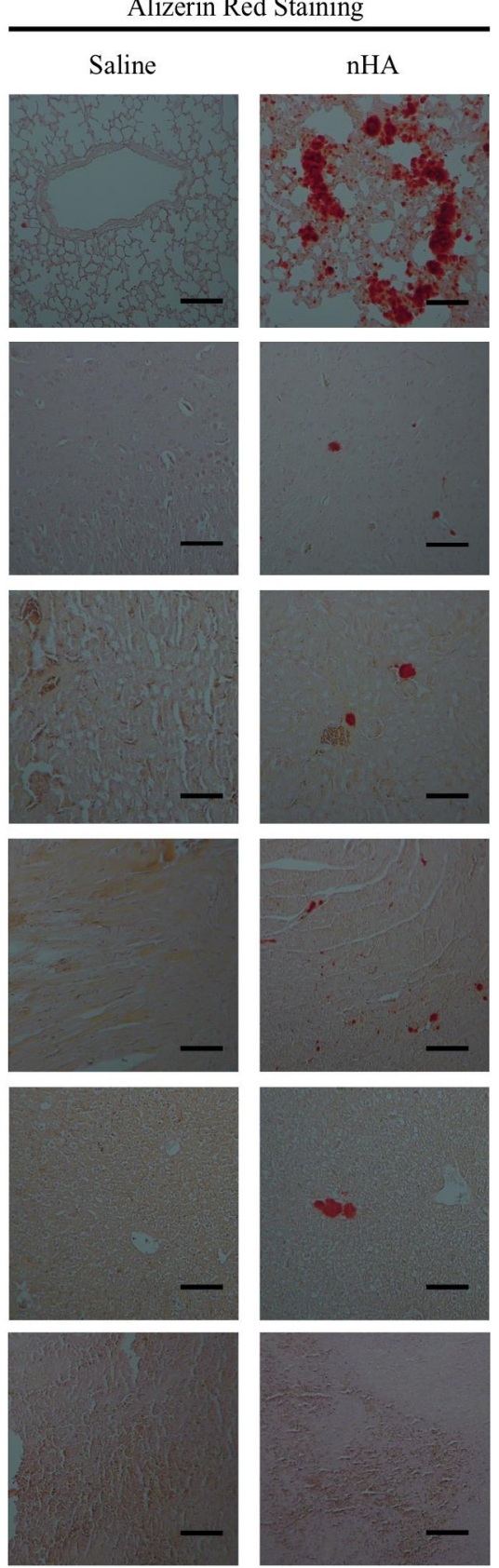

Figure 8: Tissues of BALB/c mice injected with either saline or $\mathrm{nHA}$ at $280 \mathrm{mg} \mathrm{kg}^{-1}$ were stained with haematoxylin and eosin (left) for tissue structure or Alizarin red (right) for calcium. Scale bars represent $100 \mu \mathrm{m}$.

\section{Conclusions}

This work shows a new approach for the functionalization of nHA with PGA-PEG to deliver lovastatin. PGA was grafted directly onto the surface of nHA, and PEG was attached to the chain-ends of PGA. The relatively hydrophobic PGA was able to encapsulate and control the release of lovastatin over a 2-week period. Moreover, release of lovastatin could be controlled by tailoring the thickness of the PGA coating. While nHA and nHA-PGA-PEG showed some toxicity in vitro, HUVECs showed $70 \%$ viability at doses as high as $500 \mu \mathrm{g} \mathrm{mL}^{-1}$. nHAPGA-PEG uptake was shown in mouse osteoblasts using fluorescent and differential interference microscopy. Finally, the PGA-PEG coating raised the maximum tolerated dose in $\mathrm{BALB} / \mathrm{c}$ mice when compared to uncoated nHA. This work shows that the nHA system could be used to deliver lovastatin for treatment of low-bone density pathologies.

\section{Acknowledgement}

This work was supported by SC EPSCOR-NASA 2098490, and the SC EPSCOR-NASA South Carolina Space Undergraduate Research Grant. Facilities used were supported by the South Carolina COBRE Center of Biomaterials for Tissue Regeneration (CBTR) funded under NIH Grants 5P20RR021949-04 and 8P20GM103444-04. The authors would like to thank Kim Ivey for use of the TGA and FTIR, and Dr. John Parrish and Travis Pruitt for help with the in vivo 
Citation: Moore TL, Schreurs AS, Morrison RA, Jelen EK, Loo J, et al. (2014) Polymer-Coated Hydroxyapatite Nanoparticles for the Delivery of Statins. J Nanomed Nanotechnol 5: 237. doi: 10.4172/2157-7439.1000237

work. Thomas L Moore and Ann-Sofie Schreurs have contributed equally to this manuscript.

\section{References}

1. Chesnutt BM, Yuan Y, Buddington K, Haggard WO, Bumgardner JD (2009) Composite chitosan/nano-hydroxyapatite scaffolds induce osteocalcin production by osteoblasts in vitro and support bone formation in vivo. Tissue Eng Part A 15: 2571-2579.

2. Niu X, Feng Q, Wang M, Guo X, Zheng Q (2009) Porous nano-HA/collagen/ PLLA scaffold containing chitosan microspheres for controlled delivery of synthetic peptide derived from BMP-2. J Control Release 134: 111-117.

3. Zheng F, Wang S, Wen S, Shen M, Zhu M, et al. (2013) Characterization and antibacterial activity of amoxicillin-loaded electrospun nano-hydroxyapatite/ poly(lactic-co-glycolic acid) composite nanofibers. Biomaterials 34: 1402-1412.

4. Bisht S, Bhakta G, Mitra S, Maitra A (2005) pDNA loaded calcium phosphate nanoparticles: highly efficient non-viral vector for gene delivery. Int J Pharm 288: 157-168.

5. Loo SC, Moore T, Banik B, Alexis F (2010) Biomedical applications of hydroxyapatite nanoparticles. Curr Pharm Biotechnol 11: 333-342.

6. Do TN, Lee WH, Loo CY, Zavgorodniy AV, Rohanizadeh R (2012) Hydroxyapatite nanoparticles as vectors for gene delivery. Ther Deliv 3: 623-632.

7. Ong H, Loo JC, Boey FC, Russell S, Ma J, et al. (2008) Exploiting the highaffinity phosphonate-hydroxyapatite nanoparticle interaction for delivery of radiation and drugs. J Nanopart res 10: 141-150.

8. Müller KH, Motskin M, Philpott AJ, Routh AF, Shanahan CM, et al. (2014) The effect of particle agglomeration on the formation of a surface-connected compartment induced by hydroxyapatite nanoparticles in human monocytederived macrophages. Biomaterials 35: 1074-1088.

9. Aoki H, Aoki H, Kutsuno T, Li W, Niwa M (2000) An in vivo study on the reaction of hydroxyapatite-sol injected into blood. J Mater Sci Mater Med 11: 67-72.

10. Fan Q, Wang YE, Zhao X, Loo JS, Zuo YY (2011) Adverse biophysical effects of hydroxyapatite nanoparticles on natural pulmonary surfactant. ACS Nano 5 : 6410-6416.

11. Barroug A, Glimcher MJ (2002) Hydroxyapatite crystals as a local delivery system for cisplatin: adsorption and release of cisplatin in vitro. Journal of Orthopaedic Research 20: 274-280.

12. Palazzo B, lafisco M, Laforgia M, Margiotta N, Natile G, et al. (2007) Biomimetic hydroxyapatite-drug nanocrystals as potential bone substitutes with antitumor drug delivery properties. Advanced Functional Materials 17: 2180-2188.

13. Zhou H, Wu T, Dong X, Wang Q, Shen J (2007) Adsorption mechanism of BMP7 on hydroxyapatite (001) surfaces. Biochem Biophys Res Commun 361: 91 96.

14. Kundu B, Ghosh D, Sinha Mk, Sen Ps, Balla Vk, et al. (2013) Doxorubicinintercalated nano-hydroxyapatite drug-delivery system for liver cancer: an animal model. Ceramics International 39: 9557-9566.

15. Tai IC, Fu YC, Wang CK, Chang JK, Ho ML (2013) Local delivery of controlledrelease simvastatin/PLGA/HAp microspheres enhances bone repair. Int J Nanomedicine 8: 3895-3904.

16. Cao T, Tang W, Zhao J, Qin L, Lan C (2014) A novel drug delivery carrier based on a-eleostearic acid grafted hydroxyapatite composite. Journal of Bionic Engineering 11: 125-133.

17. Morgan TT, Muddana HS, Altinoglu El, Rouse SM, TabakoviÄł A, et al. (2008) Encapsulation of organic molecules in calcium phosphate nanocomposite particles for intracellular imaging and drug delivery. Nano Lett 8: 4108-4115.

18. Yang P, Quan Z, Li C, Kang X, Lian H, et al. (2008) Bioactive, luminescent and mesoporous europium-doped hydroxyapatite as a drug carrier. Biomaterials 29: 4341-4347.

19. Nel AE, Mädler L, Velegol D, Xia T, Hoek EM, et al. (2009) Understanding biophysicochemical interactions at the nano-bio interface. Nat Mater 8: 543557

20. Choi SW, Kim JH (2007) Design of surface-modified poly(D,L-lactide-coglycolide) nanoparticles for targeted drug delivery to bone. J Control Release 122: $24-30$.

21. Ho MH, Chiang CP, Liu YF, Kuo MY, Lin SK, et al. (2011) Highly efficient release of lovastatin from poly(lactic-co-glycolic acid) nanoparticles enhances bone repair in rats. J Orthop Res 29: 1504-1510.
22. Shi X, Wang Y, Ren L, Gong Y Wang DA (2009) Enhancing alendronate release from a novel PLGA/hydroxyapatite microspheric system for bone repairing applications. Pharm Res 26: 422-430.

23. Wang S, Wang X, Xu H, Abe H, Tan Z, et al. (2010) Towards sustained delivery of small molecular drugs using hydroxyapatite microspheres as the vehicle. Advanced Powder Technology 21: 268-272.

24. Mattix B, Moore T1, Uvarov O1, Pollard S1, O'Donnell L1, et al. (2013) Effects of polymeric nanoparticle surface properties on interaction with brain tumor environment. Nano Life 3: 1343003

25. Mundy G, Garrett R, Harris S, Chan J, Chen D, et al. (1999) Stimulation of bone formation in vitro and in rodents by statins. Science 286: 1946-1949.

26. Maeda T, Kawane T, Horiuchi N (2003) Statins augment vascular endothelial growth factor expression in osteoblastic cells via inhibition of protein prenylation. Endocrinology 144: 681-692.

27. Maeda T, Matsunuma A, Kurahashi I, Yanagawa T, Yoshida H, et al. (2004) Induction of osteoblast differentiation indices by statins in MC3T3-E1 cells. J Cell Biochem 92: 458-471.

28. Wang W, Nyman JS, Moss HE, Gutierrez G, Mundy GR, et al. (2010) Local lowdose lovastatin delivery improves the bone-healing defect caused by Nf1 loss of function in osteoblasts. J Bone Miner Res 25: 1658-1667.

29. Liu X, Li X, Zhou L, Li S, Sun J, et al. (2013) Effects of simvastatin-loaded polymeric micelles on human osteoblast-like MG-63 cells. Colloids Surf B Biointerfaces 102: 420-427.

30. Yoshii T, Hafeman AE, Esparza JM, Okawa A, Gutierrez G, et al. (2014) Loca injection of lovastatin in biodegradable polyurethane scaffolds enhances bone regeneration in a critical-sized segmental defect in rat femora. Journal of Tissue Engineering and Regenerative Medicine 8: 589-595.

31. Aggarwal P, Hall JB, McLeland CB, Dobrovolskaia MA, McNeil SE (2009) Nanoparticle interaction with plasma proteins as it relates to particle biodistribution, biocompatibility and therapeutic efficacy. Adv Drug Deliv Rev 61: 428-437.

32. Perry JL, Reuter KG, Kai MP, Herlihy KP, Jones SW, et al. (2012) PEGylated PRINT nanoparticles: the impact of $P E G$ density on protein binding, macrophage association, biodistribution, and pharmacokinetics. Nano Lett 12: 5304-5310.

33. Albrektsson T, Johansson C (2001) Osteoinduction, Osteoconduction and Osseointegration. European spine journal: official publication of the European spine society, the European Spinal deformity society, and the European section of the cervical spine research society 10 suppl 2: s96-101.

34. Huang YX, Ren J, Chen C, Ren TB, Zhou XY (2008) Preparation and properties of poly(lactide-co-glycolide) (PLGA)/ nano-hydroxyapatite (NHA) scaffolds by thermally induced phase separation and rabbit MSCs culture on scaffolds. J Biomater Appl 22: 409-432.

35. Wei G, Ma PX (2004) Structure and properties of nano-hydroxyapatite/polymer composite scaffolds for bone tissue engineering. Biomaterials 25: 4749-4757.

36. LeGeros RZ (2002) Properties of osteoconductive biomaterials: calcium phosphates. Clin Orthop Relat Res : 81-98.

37. Lin L, Chow KL, Leng Y (2009) Study of hydroxyapatite osteoinductivity with an osteogenic differentiation of mesenchymal stem cells. J Biomed Mater Res A 89: $326-335$

38. Panda NN, Jonnalagadda S, Pramanik K (2013) Development and evaluation of cross-linked collagen-hydroxyapatite scaffolds for tissue engineering Journal of Biomaterials Science, Polymer Edition 24: 2031-2044.

39. Jeanmonod D, Sindou M (1991) Somatosensory function following dorsal root entry zone lesions in patients with neurogenic pain or spasticity. J Neurosurg 74: $916-932$

40. Ullman-Culleré MH1, Foltz CJ (1999) Body condition scoring: a rapid and accurate method for assessing health status in mice. Lab Anim Sci 49: 319-323.

41. Workman P, Aboagye EO, Balkwill F, Balmain A, Bruder G, et al. (2010) Guidelines for the welfare and use of animals in cancer research. $\mathrm{Br} \mathrm{J}$ Cancer 102: 1555-1577.

42. Alwood JS, Yumoto K, Mojarrab R, Limoli CL, Almeida EA, et al. (2010) Heavy ion irradiation and unloading effects on mouse lumbar vertebral microarchitecture, mechanical properties and tissue stresses. Bone 47: 248-255.

43. Blaber EA, Dvorochkin N, Lee C, Alwood JS, Yousuf R, et al. (2013) Microgravity induces pelvic bone loss through osteoclastic activity, osteocytic osteolysis, and osteoblastic cell cycle inhibition by CDKN1a/p21. PLoS One 8: e61372. 
Citation: Moore TL, Schreurs AS, Morrison RA, Jelen EK, Loo J, et al. (2014) Polymer-Coated Hydroxyapatite Nanoparticles for the Delivery of Statins. J Nanomed Nanotechnol 5: 237. doi: 10.4172/2157-7439.1000237

44. Meirelles L, Arvidsson A, Andersson M, Kjellin P, Albrektsson T, et al. (2008) Nano hydroxyapatite structures influence early bone formation. J Biomed Mater Res A 87: 299-307.

45. Koutsopoulos S (2002) Synthesis and characterization of hydroxyapatite crystals: a review study on the analytical methods. J Biomed Mater Res 62: 600-612.

46. Liu Q, de Wijn JR, de Groot K, van Blitterswijk CA (1998) Surface modification of nano-apatite by grafting organic polymer. Biomaterials 19: 1067-1072.

47. Kim SY, Shin IG, Lee YM (1999) Amphiphilic diblock copolymeric nanospheres composed of methoxy poly(ethylene glycol) and glycolide: properties, cytotoxicity and drug release behaviour. Biomaterials 20: 1033-1042.

48. Alexis $F(2005)$ Factors affecting the degradation and drug-release mechanism of poly(lactic acid) and poly[(lactic acid)-co-(glycolic acid)]. Polymer International 54: 36-46.

49. Lao LL, Peppas NA, Boey FY, Venkatraman SS (2011) Modeling of drug release from bulk-degrading polymers. Int J Pharm 418: 28-41.
50. Moore TL, Podilakrishna R, Rao A, Alexis F (2014) Systemic administration of polymer-coated nano-graphene to deliver drugs to glioblastoma. Particle \& Particle Systems Characterization 31: 886-894.

51. Chen CH, Yang JC, Uang YS, Lin CJ (2013) Improved dissolution rate and oral bioavailability of lovastatin in red yeast rice products. Int J Pharm 444: 18-24.

52. Motskin M, Wright DM, Muller K, Kyle N, Gard TG, et al. (2009) Hydroxyapatite nano and microparticles: correlation of particle properties with cytotoxicity and biostability. Biomaterials 30: 3307-3317.

53. Zhao X, Heng BC, Xiong S, Guo J, Tan TT, et al. (2011) In vitro assessment of cellular responses to rod-shaped hydroxyapatite nanoparticles of varying lengths and surface areas. Nanotoxicology 5: 182-194.

54. Tan JS, Butterfield DE, Voycheck CL, Caldwell KD, Li JT (1993) Surface modification of nanoparticles by PEO/PPO block copolymers to minimize interactions with blood components and prolong blood circulation in rats. Biomaterials 14: 823-833.

55. Owens DE 3rd, Peppas NA (2006) Opsonization, biodistribution, and pharmacokinetics of polymeric nanoparticles. Int J Pharm 307: 93-102. 